\title{
HYBRID ESTIMATES OF PATH ATTENUATION FOR THE DPR
}

\author{
Robert Meneghini ${ }^{1}$, Liang Liao $^{2}$, Toshio Iguchi ${ }^{3}$ and Hyokyung Kim $^{2}$
}

1. NASA/Goddard Space Flight Center, Code 612, Greenbelt, MD, 20771

2. Goddard Earth Science Technology and Research, Code 612, Greenbelt, MD 20771

3. National Institute of Information and Communications Technology (NICT), Tokyo 184-8795, Japan

\begin{abstract}
The purpose of this study is to investigate ways to combine estimates from the Surface Reference and Hitschfeld-Bordan methods into a hybrid path attenuation estimate and to study its performance using dual-frequency radar data provided by the DualFrequency Precipitation Radar (DPR) on board the Global Precipitation Mission (GPM) satellite.
\end{abstract}

Index Terms - path attenuation, DPR, weather radar

\section{INTRODUCTION}

For airborne or spaceborne weather radars operating at frequencies of $10 \mathrm{GHz}$ and above, estimation of and correction for attenuation is a basic part of any rain retrieval method. Two well-known ways of obtaining a path attenuation estimate are the Hitschfeld-Bordan (HB) [1] and the Surface Reference (SRT) [2] techniques. Both methods suffer from a variety of errors. In the case of the HB, the estimate becomes unstable at high values of path attenuation; errors in the radar calibration or in the selection of the $\mathrm{k}-\mathrm{Z}$ (specific attenuation-radar reflectivity factor) tend to magnify the error. For the SRT, which relies upon the stability of the surface cross section within and outside the rain, errors occur when the rain-free surface cross section differs from that within the raining area. Generally speaking, with an increase in path attenuation, the relative error in the $\mathrm{HB}$ increases while the relative error in the SRT decreases. This implies that as the radar operating frequency or rain rate is increased, both of which lead to higher path attenuations, the retrieval algorithm should favor the SRT over the HB; conversely, for lighter rain rates and lower frequencies, the HB should generally be employed.

\section{SRT AND HB PATH ATTENUATIONS}

For the SRT, the path integrated attenuation (PIA) estimate, $A(d B)$, at radar frequency $\mathrm{f}$, is obtained by subtracting the normalized surface cross section, $\sigma^{0}$, in rain (R) from a reference measurement of $\sigma^{0}$ taken outside the rain (NR). This can be written [3]

$$
A_{S R T}(f)=\sigma_{N R}^{0}(f)-\sigma_{R}^{0}(f)
$$

With two frequencies, an estimate for the differential path attenuation $\left(\delta A=A\left(f_{1}\right)-A\left(f_{2}\right)\right)$ where $f_{1}$ is taken to be the higher, more attenuated frequency (which in our case is the Ka-band frequency), can be written [3]:

$\delta A_{S R T}=\delta \sigma_{N R}^{0}-\delta \sigma_{R}^{0}$

It has been shown that the errors in (2) tend to be smaller than those associated with (1) because the surface cross sections at $\mathrm{Ku}$ and Ka-band tend to be highly correlated; in particular, the variance of $\delta \sigma 0$ in rain-free conditions tends to be smaller than the variance of $\sigma^{0}(\mathrm{Ku})$ or $\sigma^{0}(\mathrm{Ka})$ and, except for nadir and near-nadir incidence over land, is relatively independent of the radar incidence angle. For the HB, the estimate of path attenuation can be expressed as

$$
A_{H B}(f)=-(10 / \beta(f)) \log _{10}(1-\zeta(f))
$$

where

$$
\zeta(f)=0.2 \ln (10) \beta \int_{0}^{r_{s}} \alpha(s) Z_{m}^{\beta}(s) d s
$$

and where the limits of integration are from the storm top to the surface. The quantities $\alpha$ and $\beta$ are the parameters in the $\mathrm{k}-\mathrm{Z}$ (specific attenuation-radar reflectivity factor) relationship, $\mathrm{k}=\alpha Z^{\beta}$. Finally, the 
measured, $\mathrm{Z}_{\mathrm{m}}$, and actual, $\mathrm{Z}$, radar reflectivity factors are related by

$$
Z(f)=Z_{m}(f) \exp \left[0.2 \ln (10) \int_{0}^{r_{s}} k(s, f) d s\right]
$$

The expression for differential attenuation, using the HB method, is obtained simply by applying the above formula to each frequency and subtracting:

$$
\delta A_{H B}=10 \log _{10}\left\{\left[1-\zeta\left(f_{2}\right)\right]^{1 / \beta_{2}} /\left[1-\zeta\left(f_{1}\right)\right]^{1 / \beta_{1}}\right\}(6)
$$

\section{HYBRID PATH ATTENUATIONS}

A simple way to merge the SRT and HB estimates of path attenuation is to use the formula

$$
\begin{aligned}
& A_{H Y}(f)=n\left[A_{S R T}(f) / \sigma_{S R T}^{2}+A_{H B}(f) / \sigma_{H B}^{2}\right] \\
& \text { where } n=1 /\left[1 / \sigma_{S R T}^{2}+1 / \sigma_{H B}^{2}\right]
\end{aligned}
$$

where ' $\mathrm{HY}$ ' denotes the hybrid estimate and $\sigma_{\mathrm{SRT}}$ and $\sigma_{\mathrm{HB}}$ are the standard deviations associated with the $\mathrm{A}_{\mathrm{SRT}}$ and $\mathrm{A}_{\mathrm{HB}}$ estimates, respectively. Similarly, for the differential-frequency estimate, we can write

$$
\delta A_{H Y}=\left[\delta A_{S R T} / \sigma_{\delta S R T}^{2}+\delta A_{H B} / \sigma_{\delta H B}^{2}\right] /\left[1 / \sigma_{\delta S R T}^{2}+1 / \sigma_{\delta H B}^{2}\right](8)
$$

where $\sigma_{\delta \mathrm{SRT}}$ and $\sigma_{\delta \mathrm{HB}}$ are the standard deviations associated with the $\delta A_{\mathrm{SRT}}$ and $\delta \mathrm{A}_{\mathrm{HB}}$ estimates, respectively.

It is worth noting that the equations above provide a minimum variance estimate given two independent estimates of path attenuation with their associated variances. A general formula of this type for $\mathrm{N}$ independent random variables can be derived by the method of Lagrange multipliers [4].

A difficulty with the formulation arises from the fact that the variances are generally assumed to be fixed and independent of the magnitude of the estimate. This is not the case for the HB estimate where, according to (3), the result is unbounded as $\zeta$ approaches unity. As discussed below, to deal with this problem requires that the variances of $A_{\mathrm{HB}}$ and $\delta A_{\mathrm{HB}}$ be expressed as a function of $\zeta$.

Merging of the HB and SRT estimates was first given in [5] and later in [6, 7]. Following the approach in
[6] and using the notation given here, the following equation is minimized:

$$
\begin{aligned}
& D \equiv\left(A-A_{S R T}\right)^{2} / 2 \sigma_{S R T}^{2}+ \\
& \{\ln (\zeta)-\ln [1-\exp (-0.1 \ln (10) \beta A)]\}^{2} / 2 \sigma_{\ln \zeta}^{2}
\end{aligned}
$$

where $\sigma_{\ln \zeta}^{2}$ is the variance associated with $\ln (\zeta)$. Notice that the numerator of the second term of this equation can be derived by rewriting (3) as a function of $\ln (\zeta)$ and letting $A_{\mathrm{HB}}=\mathrm{A}$. Taking the derivative of $\mathrm{D}$ with respect to $\mathrm{A}$ and setting the result to zero yields a transcendental equation for $\mathrm{A}$ that can be solved numerically. Although preliminary results obtained from (9) seem to be comparable to those obtained from (7), there is no obvious analog for the dualfrequency situation. On the other hand, the variance of $\ln (\zeta)$ is much easier to specify than the variance of $A_{H B}$ as this latter variance is a function of $\zeta$ and path length. Because of the merits and demerits of the two approaches, we retain both for purposes of understanding their respective strengths and weaknesses.

As noted above, a critical factor in all formulations is the accurate specification of the variances. For the variance of $\ln (\zeta)$, we first write $\alpha=\varepsilon \alpha_{0}$ where $\alpha_{0}$ is taken to be the mean value and $\log (\varepsilon)$ a zero-mean random variable that characterizes changes in the $\mathrm{k}-\mathrm{Z}$ relationship caused by fluctuations in the raindrop size distribution. Using these relationships, and eq. (4), we write

$$
\ln (\zeta) \approx \ln (\varepsilon)+\ln \left[0.2 \ln (10) \beta \alpha_{0} \int_{0}^{r_{s}} Z_{m}^{\beta}(s) d s\right]
$$

Assuming that the second term is approximately constant, then to a first approximation $\operatorname{var}(\ln (\zeta))=\operatorname{var}(\ln (\varepsilon))$. Shown in Fig. 1 is the distribution of $\varepsilon$ at Ku-band as derived from measured raindrop size distributions (DSD). (Basically, from each DSD the corresponding $\mathrm{k}$ and $\mathrm{Z}$ are computed; then, after fixing $\beta$, the distribution of $\alpha$ is derived.) From the figure we find that the standard deviation, $\sigma$, of $\log _{10} \varepsilon$ is approximately 0.18 so that $\sigma(\ln \varepsilon)=0.414$. We find, moreover, that the distribution and statistics of $\varepsilon$ at Ka-band are nearly identical to those at Kuband. 


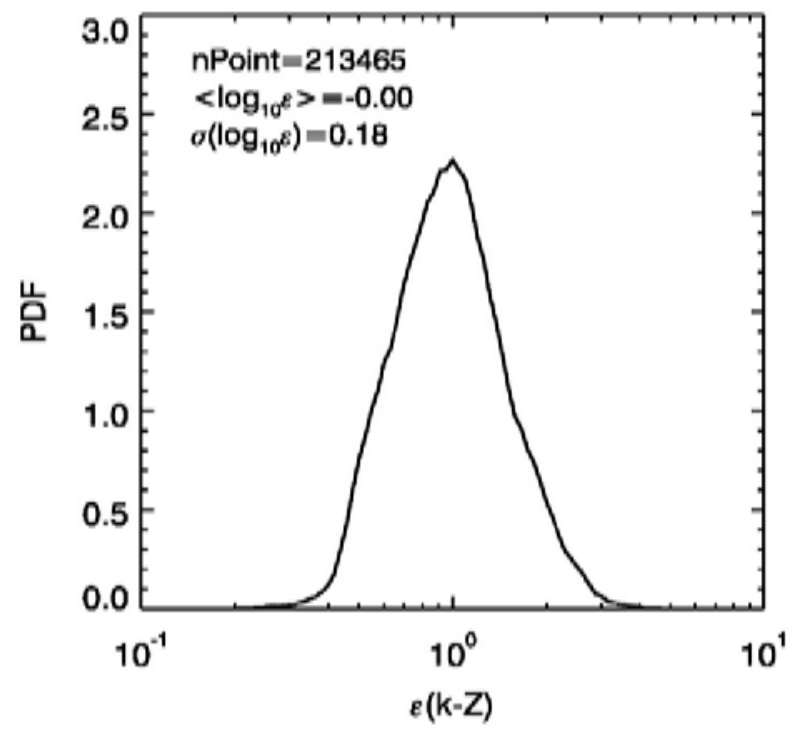

Fig. 1. Distribution of $\varepsilon$ where $\alpha=\varepsilon \alpha_{0}$ as derived from measured raindrop size distributions.

Specification of $\sigma_{S R T}^{2}$ is somewhat complex and has been discussed elsewhere [3]. We note, however, that $A_{\text {SRT }}$ and $\delta A_{\text {SRT }}$ are themselves estimated as the weighted sums of as many as 5 independent estimates of the SRT. These different estimates arise from the fact that different rain-free surface reference estimates can be made, e.g., from along and cross track rain-free data processed in the forward/backward direction and from prior rain-free data at the same location as the raining pixel. As these estimates are nearly independent of each other, the variance of the weighted sum is given approximately by

$\sigma_{\text {eff }}^{2}=\left[\sum 1 / \sigma_{j}^{2}\right]^{-1}$

where $\sigma_{j}^{2}$ is the variance of the jth SRT-PIA estimate.

To this term has been added a second contribution to the variance that is basically equal to the mean-square deviation of the individual PIA-SRT estimates from the effective or final PIA.

The final terms that must be specified are the variances associated with $\mathrm{A}_{\mathrm{HB}}$ and $\delta \mathrm{A}_{\mathrm{HB}}$. Shown in Fig. 2 are the mean and standard deviations for $\mathrm{A}_{\mathrm{HB}}(\mathrm{Ku}), \mathrm{A}_{\mathrm{HB}}(\mathrm{Ka})$ and $\delta \mathrm{A}_{\mathrm{HB}}$ as a function of $\zeta$ for a path length of $3.5 \mathrm{~km}$. Note that $\zeta=\zeta(\mathrm{Ka})$ for the $\mathrm{A}(\mathrm{Ka})$ and $\delta \mathrm{A}$ curves but $\zeta=\zeta(\mathrm{Ku})$ for the $\mathrm{A}(\mathrm{Ku})$. The results show that the standard deviation (center panel)
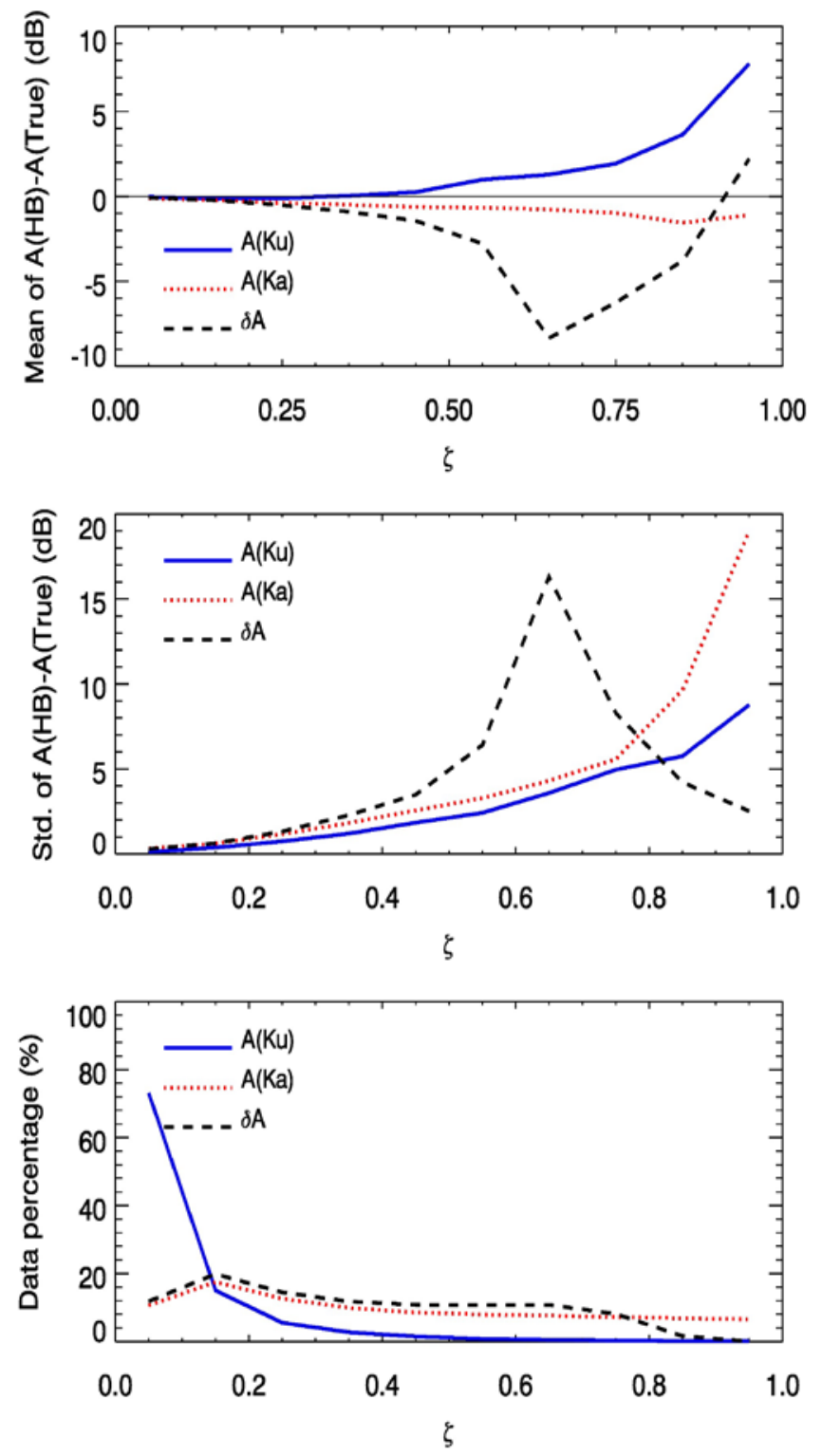

Fig. 2. Mean (top) and standard deviation (center) of the HB path attenuation estimates as derived from measured drop size distributions. Results in the bottom panel show the percentage of points using a 0.1 bin size. For example, about $75 \%$ of the Ku-band data have $\zeta$ values between 0 and 0.1 .

increases uniformly with $\zeta$ for $\mathrm{Ku}$ - and Ka-band but reaches a maximum at about $\zeta=0.65$ for the $\mathrm{Ka} / \mathrm{Ku}$ difference.

\section{PRELIMINARY RESULTS}

Preliminary results are shown in Fig. 3 using DPR data from orbit 1778 taken on 21 June 2014. Because 


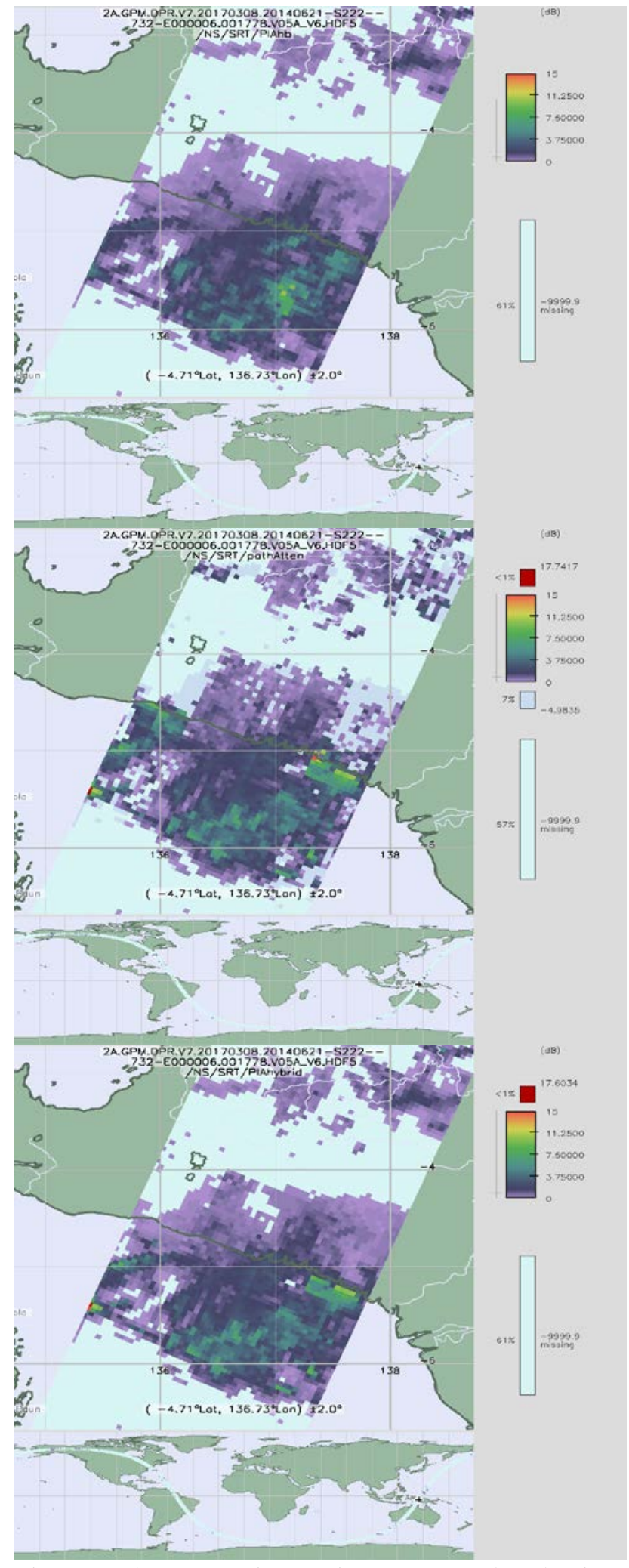

Fig. 3. Path Attenuation Estimates at Ku-band using dual-frequency radar data: HB (top), SRT (center) and Hybrid (bottom). of space limitations, only the dual-frequency retrievals are shown, where for all methods, we use: $A(K u)=$ $0.2 * \delta \mathrm{A}$, which assumes that $\mathrm{A}(\mathrm{Ka}) / \mathrm{A}(\mathrm{Ku})=6$. Note that, at least in a qualitative sense, the hybrid estimates behave as expected where the HB-PIA dominates at the lighter PIA values while the SRT-PIA dominates in regions of higher PIA. For the results shown, we have used (8) but indications are that the standard method, where the hybrid procedure is done separately at each frequency, produces similar results.

\section{SUMMARY}

An important issue in attenuation correction methods for the DPR is merging the estimates from the SRT and $\mathrm{HB}$ in an optimal way. Examination of the traditional and an alternative method shows that both appear to provide a means to merge the SRT and HB estimates for both single- and dual-frequency attenuation estimates. Additional theoretical work and testing with the DPR data will be needed to determine which approach leads to the most accurate path attenuation estimate.

\section{REFERENCES}

[1] W. Hitschfeld and J. Bordan, "Errors inherent in the radar measurement of rainfall at attenuating wavelengths", J. Meteor., Vol. 11, pp. 58-67.

[2] R. Meneghini, J. Eckerman, and D. Atlas, "Determination of Rain Rate from a Spaceborne Radar using Measurements of Total Attenuation”, IEEE Trans. GeoSci. Remote Sens., Vol. GE-21, pp. 34-43, 1983.

[3] R. Meneghini, et al., "An initial assessment of the Surface Reference Technique applied to data from the DualFrequency Precipitation Radar (DPR) on the GPM Satellite”, J. Atmos. Oceanic Technol., Vol 32, pp. 22812296, 2015.

[4] R. Daley, Atmospheric Data Analysis, Cambridge University Press, 1991.

[5] T. Iguchi and R. Meneghini, "Intercomparisons of single-frequency methods for retrieving a vertical rain profile from airborne or spaceborne radar data”, J. Atmos. Oceanic Technol, Vol 11, pp. 1507-1516, 1994.

[6] T. Iguchi, et al., Rain-Profiling Algorithm for the TRMM Precipitation Radar, J. Appl. Meteor., Vol 39, pp. 20382052, 2000.

[7] T. Iguchi et al., "Uncertainties in the Rain Profiling Algorithm for the TRMM Precipitation Radar", J. Meteor. Soc. Japan, Vol. 87A, pp. 1-30, 2009. 